\title{
Gustatory Receptors Required for Avoiding the Insecticide L-Canavanine
}

\author{
Youngseok Lee, ${ }^{1 \star}$ Min Jung Kang, ${ }^{2 \star}$ Jaewon Shim, ${ }^{2}$ Chae Uk Cheong, ${ }^{2}$ Seok Jun Moon, ${ }^{2}$ and Craig Montell ${ }^{1}$ \\ ${ }^{1}$ Department of Biological Chemistry and Department of Neuroscience, Center for Sensory Biology, The Johns Hopkins University School of Medicine, \\ Baltimore, Maryland 21205, and 2Department of Oral Biology, Brain Korea 21 Project, Yonsei University College of Dentistry, Seodaemun-gu, Seoul 120 - \\ 752 , Korea
}

Insect survival depends on contact chemosensation to sense and avoid consuming plant-derived insecticides, such as L-canavanine. Members of a family of $\sim 60$ gustatory receptors (GRs) comprise the main peripheral receptors responsible for taste sensation in Drosophila. However, the roles of most Drosophila GRs are unknown. In addition to GRs, a G protein-coupled receptor, DmXR, has been reported to be required for detecting L-canavanine. Here, we showed that GRs are essential for responding to L-canavanine and that flies missing DmXR displayed normal L-canavanine avoidance and L-canavanine-evoked action potentials. Mutations disrupting either Gr8a or Gr66 a resulted in an inability to detect $\mathrm{L}$-canavanine. We found that $\mathrm{L}$-canavanine stimulated action potentials in S-type sensilla, which were where Gr8a and Gr66a were both expressed, but not in Gr66a-expressing sensilla that did not express Gr8a. L-canavanine-induced action potentials were also abolished in the Gr8a and Gr66a mutant animals. Gr8a was narrowly required for responding to L-canavanine, in contrast to Gr66a, which was broadly required for responding to other noxious tastants. Our data suggest that GR8a and GR66a are subunits of an L-canavanine receptor and that GR8a contributes to the specificity for L-canavanine.

\section{Introduction}

Contact chemosensation is crucial for animals to discriminate safe, nutritious foods from toxic substances in the environment. In insects such as the fruit fly, Drosophila melanogaster, this ability is essential for survival since many plants produce compounds with insecticidal properties. One such botanically derived insecticide is L-canavanine, which is an analog of the amino acid L-arginine (Dahlman and Rosenthal, 1975; Rosenthal and Dahlman, 1986; Rosenthal, 2001). Consumption of L-canavanine causes lethality to fruit flies and many other insects, since this amino acid derivative is incorporated into proteins in place of L-arginine, thereby hindering protein function.

In fruit flies, tastants are detected in gustatory receptor neurons (GRNs), which are housed in gustatory hairs (sensilla) (Vosshall and Stocker, 2007; Montell, 2009). The main class of taste receptors consists of $\sim 60$ gustatory receptors (GRs), and these multipass transmembrane proteins are unrelated to $G$

\footnotetext{
Received Sept. 10, 2011; revised Nov. 18, 2011; accepted Dec. 6, 2011

Author contributions: Y.L., M.J.K., S.J.M., and C.M. designed research; Y.L., M.J.K., J.S., C.U.C., and S.J.M. performed research; Y.L., M.J.K., S.J.M., and C.M. analyzed data; S.J.M. and C.M. wrote the paper.

This work was supported by a grant to C.M. from the NIDCD (DC007864) and by grants to S.J.M. from the Converging Research Center Program funded by the Ministry of Education, Science, and Technology (2011K000678) and from the Basic Science Research Program through the National Research Foundation of Korea funded by the Ministry of Education, Science, and Technology (2010-0016576). We thank T. Sokabe for examining the function of GRs in vitro and J. Kim, Y. Grau, K. Scott, and J. Carlson for fly stocks.

*Y.L. and M.J.K. contributed equally to this work.

Correspondence should be addressed to either of the following: Seok Jun Moon, Department of Oral Biology, Brain Korea 21 Project, Yonsei University College of Dentistry, 250 Seongsanno, Seodaemun-gu, Seoul 120-752, Korea, E-mail: sjmoon@yuhs.ac; or Craig Montell, Department of Biological Chemistry and Neuroscience, Center for Sensory Biology, The Johns Hopkins University School of Medicine, 855 North Wolfe Street, Baltimore, MD 21205, E-mail: cmontell@jhmi.edu.

DOI:10.1523/JNEUROSCI.4630-11.2012

Copyright $\odot 2012$ the authors $\quad 0270-6474 / 12 / 321429-07 \$ 15.00 / 0$
}

protein-coupled receptors (GPCRs) (Clyne et al., 2000; Robertson et al., 2003; Montell, 2009). Currently, only four GRs (GR32a, GR33a, GR66a, and GR93a) are known to be required for sensing aversive tastants, ranging from caffeine to quinine, strychnine, and the synthetic repellent DEET (Moon et al., 2006, 2009; Lee et al., 2009, 2010). In contrast to the fructose receptor (GR43a) (Sato et al., 2011) and possibly the glycerol receptor (GR64e) (Wisotsky et al., 2011), the subunit composition of GRs that respond to sugars (Dahanukar et al., 2007; Jiao et al., 2007, 2008) and to aversive chemicals is complex and appears to consist of at least three subunits (Lee et al., 2009; Moon et al., 2009). The TRPA1 channel as well as GRs are expressed in GRNs and respond to noxious compounds (Kang et al., 2010; Kim et al., 2010). In addition to GRs and TRP channels, a GPCR, DmXR, encoded by the mangetout $(m t t)$ gene, has been reported to be required for the behavioral avoidance to L-canavanine (Mitri et al., 2009).

Here, we found that both the behavioral and electrophysiological responses to L-canavanine were indistinguishable between mtt mutant and control flies. Rather, Gr8a and Gr66 $a$ were indispensible for sensing L-canavanine. While Gr66a was required for detecting multiple aversive tastants (Moon et al., 2006; Lee et al., 2010), Gr8a mutant flies responded normally to all other repellent compounds tested. We propose that GRs that function in the sensation of aversive tastants are comprised of assemblies consisting of a narrowly tuned subunit, as well as at least one broadly required coreceptor.

\section{Materials and Methods}

Generation of the $\mathrm{Gr} \mathrm{a}^{1}$ mutant and transgenic flies. The P-element line G17495, which was inserted in the Gr8a coding region, was donated by the Drosophila Library Facility Biomedical Research Center (Korea Ad- 
vanced Institute of Science and Technology, Daejeon, South Korea). To generate the Gr8a ${ }^{1}$ mutant, the P-element was mobilized by genetically introducing transposase using the $\mathrm{P}[r y(\Delta 2-3)]$ line. The $\Delta 2-3$ transgene results in a high level of transposase expression as a result of deleting an intron that is normally spliced out inefficiently (Laski et al., 1986). To identify a deletion that removed Gr8a, we conducted a PCR-based screen using the following primers: P1 (TCCGAAACATACAGTGGC GTCGAT) andP2(TGTTCTTCGTGCTGACTG GCTACA) (Fig. 2A,B). We recovered one P-element excision line out of 200 lines screened. The deletion was confirmed by genomic PCR using the following primers: P2 and P3 (GCTCAAATGTTTCCAGGGTGCCTT) (Fig. $2 A, B$ ). To determine the ends of the deletion, we amplified the genomic DNA and obtained the DNA sequence. The deletion was 934 base pairs and removed the sequences encoding the amino acid residues $15-305$.

To obtain P[UAS-Gr8a] and P[UAS-Gr66a] transgenic flies, we prepared RNA from the labellum of wild-type flies and amplified the Gr8a and Gr66a cDNA by RT-PCR. We subcloned the cDNA into the pUAST vector (Brand and Perrimon, 1993). The transformation vector was injected into $w^{1118}$ embryos.

Fly stocks. We reported the following mutants previously and deposited them with the Bloomington Stock Center: Gr33a $a^{G A L 4}$ (Moon et al., 2009), Gr66 ${ }^{\text {ex83 }}$ (Moon et al., 2006), and $\mathrm{Gr} 93 a^{3}$ (Lee et al., 2009). mtt fo6268 and $\operatorname{Df}(2 \mathrm{R})$ Exel7096 were obtained from Y. Grau (University of Montpellier, Montpellier, France; Mitri et al., 2009). H. Amrein (Texas A\&M Health Science Center, College Station, TX) provided the Gr32a $a^{l}$ (Miyamoto and Amrein, 2008) and the $\mathrm{P}[$ Gr66a-GAL4] flies (Thorne et al., 2004). The P[Gr8a-GAL4] flies and $\mathrm{P}[$ Gr66a-I-GFP] were from J. Carlson (Yale University, New Haven, CT; Weiss et al., 2011) and K. Scott (University of California, Berkeley, CA; Wang et al., 2004), respectively. The fly stocks were outcrossed for at least five generations into a $w^{1118}$ background, and $w^{1118}$ was used as the "wild-type" control.

Immunohistochemistry. The labella of GR8a-GAL4/+; UAS-DsRed/ GR66a-I-GFP flies were dissected and fixed using 4\% paraformaldehyde with $0.2 \%$ Triton X-100 for $15 \mathrm{~min}$ at room temperature. The labella were washed three times with PBST $(1 \times$ PBS and $0.2 \%$ Triton X-100), cut in half with a razor blade, and blocked with $0.5 \%$ goat serum in PBST for $30 \mathrm{~min}$ at room temperature. The labella were transferred to new blocking buffer containing the primary antibodies and incubated overnight at $4^{\circ} \mathrm{C}$ : mouse anti-GFP (Molecular Probe, 1:1000) or rabbit anti-DsRed (Clontech, 1:1000). The labella were washed three times with PBST at room temperature and incubated with secondary antibodies (goat anti-mouse Alexa Fluor 488 and goat anti-rabbit Alexa Fluor 568) for $1 \mathrm{~h}$ at room temperature. The tissues were washed three times with PBST and mounted in VECTASHELD (Vector Laboratories). The samples were viewed using a Carl Zeiss LSM510 confocal microscope. The sensilla expressing Gr8a were identified by examining the dendrites of the GRNs in the fluorescent image and the bristles by differential interference contrast imaging.

Chemicals. Sucrose, caffeine, L-canavanine, DEET, denatonium, lobeline, papaverine, quinine, strychnine, L-arginine and $\mathrm{N}$-methyl-L-arginine (NMA), sulforhodamine $\mathrm{B}$, erioglaucine, and $\mathrm{KCl}$ were purchased from Sigma-Aldrich. Berberine sulfate trihydrate and Brilliant Blue FCF were obtained from Wako Pure Chemical Industries.
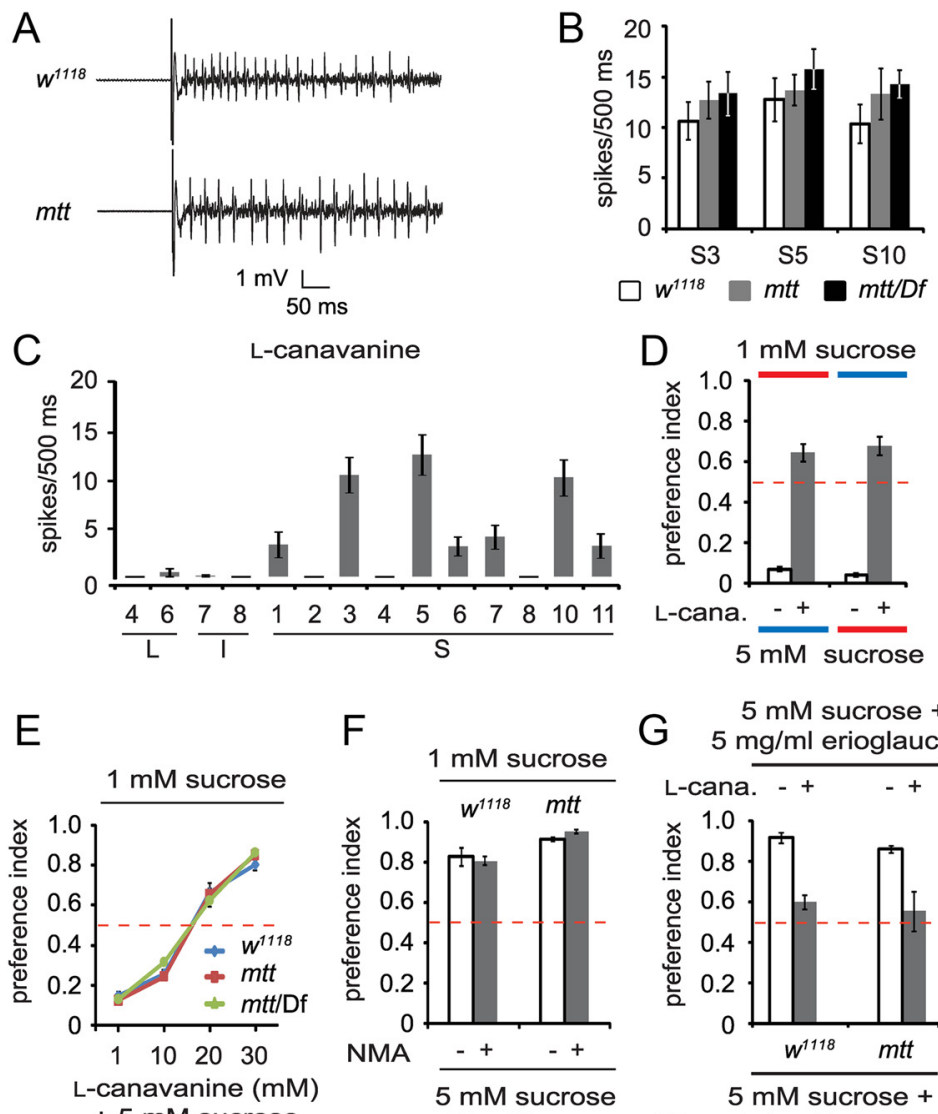

$\mathrm{F}$
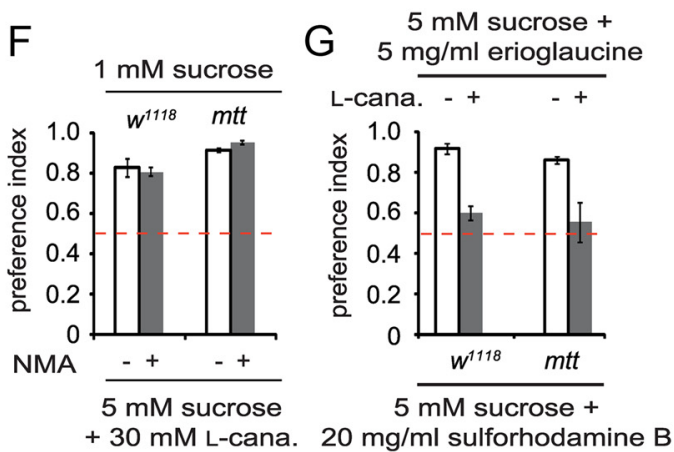

Figure 1. mtt was not required for L-canavanine-induced action potentials or for behavioral avoidance. $\boldsymbol{A}$, Representative traces L-canavanine. $\boldsymbol{B}$, Mean number of action potentials in response to $25 \mathrm{~mm} \mathrm{~L}$-canavanine recorded from S3, S5, and S10 sensilla. The 作 food dyes on two-way choice assays. The flies were given a choice between $1 \mathrm{~mm}$ sucrose and $5 \mathrm{~mm}$ sucrose (plus or minus $30 \mathrm{~mm}$ the selection of 5 mu versus 1 mm sucrose; $n=4-10$ for each concentration. Shown are means $+S E M s$. $F$ Two-way choice tests in the presence or absence of $30 \mathrm{~mm}$ NMA. G, Two-way choice tests using $5 \mathrm{~mm}$ sucrose plus $5 \mathrm{mg} / \mathrm{ml}$ erioglaucine (with or without $30 \mathrm{~mm}$ L-canavanine) versus $5 \mathrm{~mm}$ sucrose plus $20 \mathrm{mg} / \mathrm{ml}$ sulforhodamine $B$.

Two-way choice behavioral assays. With the exception of the data shown in Figure $1 G$ and Figure $2 E$, the two-way choice assays were conducted as described previously (Meunier et al., 2003; Moon et al., 2006). Briefly, we starved 50 flies (3-6 d old) for $24 \mathrm{~h}$ and then introduced the animals into 72 -well microtiter dishes. Alternating wells were filled with $1 \%$ agarose combined with one of two types of test mixtures: $1 \mathrm{~mm}$ sucrose or $5 \mathrm{~mm}$ sucrose plus an avoidance chemical. To monitor food intake, one test mixture contained a blue dye (Brilliant Blue FCF, 0.125 $\mathrm{mg} / \mathrm{ml}$ ) while the other contained a red dye (sulforhodamine B, $0.2 \mathrm{mg} /$ $\mathrm{ml}$ ). After allowing the flies to feed for $90 \mathrm{~min}$ at room temperature in the dark, the animals were frozen at $-20^{\circ} \mathrm{C}$. The numbers of flies that were blue $\left(N^{\mathrm{B}}\right)$, red $\left(N^{\mathrm{R}}\right)$, or purple $\left(N^{\mathrm{MIX}}\right)$ were determined in a blind fashion based on the colors of the abdomen, and the preference index (P.I.) values were calculated according to one of the two following equations: $\left(N^{\mathrm{B}}+0.5 N^{\mathrm{mix}}\right) /\left(N^{\mathrm{R}}+N^{\mathrm{B}}+N^{\mathrm{mix}}\right)$ or $\left(N^{\mathrm{R}}+0.5 N^{\text {mix }}\right) /\left(N^{\mathrm{R}}+N^{\mathrm{B}}+\right.$ $N^{\text {mix }}$, depending on the dye/tastant combinations. P.I.s equal to 1.0 and 0 indicated complete preferences for either the 1 or 5 mm sucrose, respectively. A P.I. equal to 0.5 indicated no preference between the two food alternatives.

To test further a possible role for $m t t$ in L-canavanine avoidance, we also performed two-way choice behavioral assays as described previously (Mitri et al., 2009). In these assays (Fig. $1 G$ and $2 E$ ), we added $5 \mathrm{~mm}$ 
A

G17495

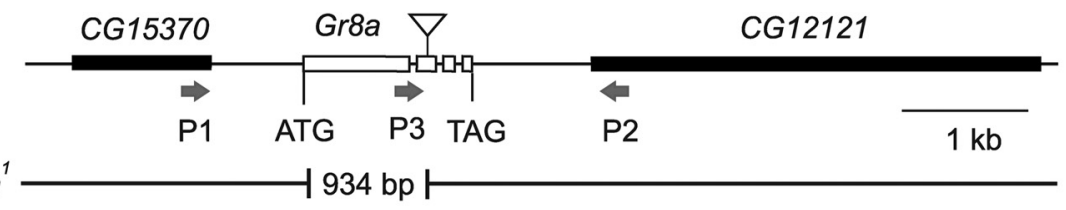
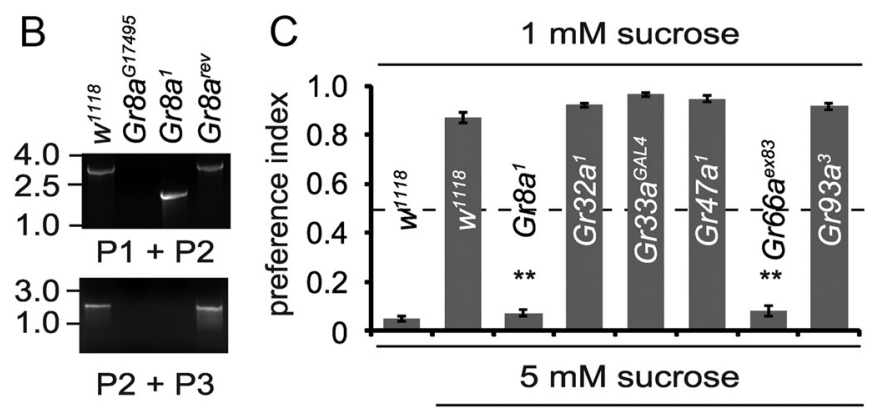

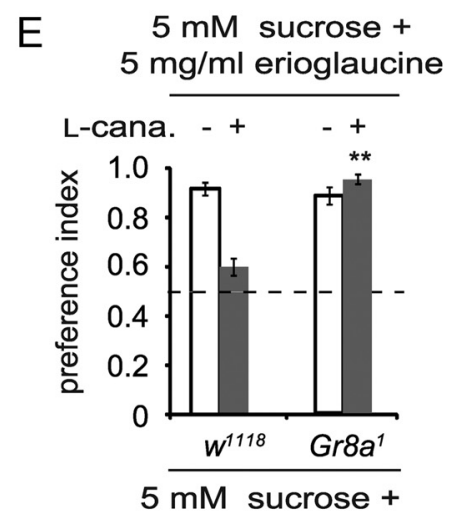

$20 \mathrm{mg} / \mathrm{ml}$ sulforhodamine $B$

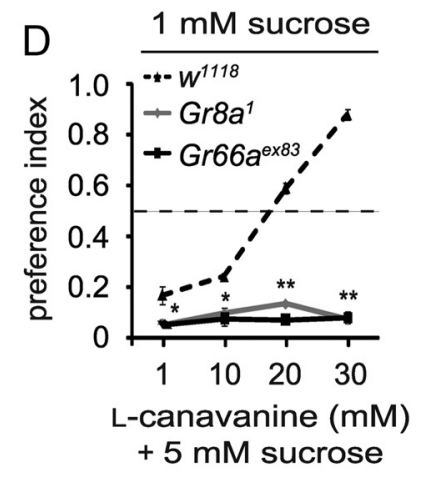

30 mM L-canavanine
$1 \mathrm{mM}$ sucrose

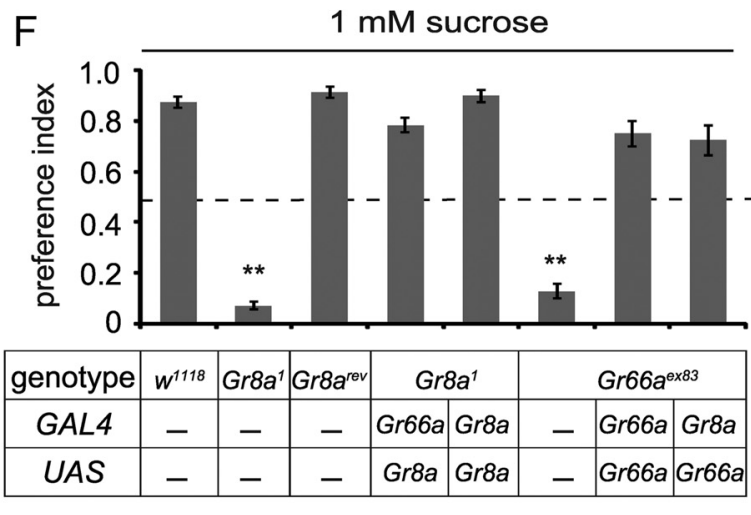

$5 \mathrm{mM}$ sucrose $+30 \mathrm{mM}$ L-canavanine

Figure 2. GRs required for L-canavanine avoidance. A, Physical map of the Gr8a genomic region. The P-element (G17495) that inserted into the Gr8a coding region is indicated by the inverted triangle. The deletion and precise excision were confirmed using the indicated PCR primers (arrows) to amplify genomic DNA (P1, P2, and P3), followed by DNA sequencing. $\boldsymbol{B}$, PCR analyses of genomic DNA. The PCR products were generated using the indicated fly stocks and PCR primer pairs and fractionated on an agarose gel. C, Screen for Grs required for $\mathrm{L}-\mathrm{canavanine}$ avoidance. The two-way choice tests were performed using the indicated chemicals and fly stocks; $n=5-15$. D, Dose-avoidance relationship determined using the two-way choice behavioral assay. Flies of the indicated genotypes were tested using L-canavanine over a range of concentrations (1-30 mm); $n=4-11$. E, Two-way choice assays using $5 \mathrm{~mm}$ sucrose plus $5 \mathrm{mg} / \mathrm{ml}$ erioglaucine (in the presence or absence of $30 \mathrm{~mm} \mathrm{~L}$-canavanine) versus $5 \mathrm{~mm}$ sucrose plus $20 \mathrm{mg} / \mathrm{ml}$ sulforhodamine B. $\boldsymbol{F}$, Rescue of L-canavanine avoidance defects by expressing wild-type Gr transgenes in GRNs using the GAL4/UAS system; $n=6-13$. The error bars indicate SEMs; ${ }^{*} p<0.05,{ }^{* *} p<0.01$.

sucrose to all wells in the microtiter dishes. Alternating wells also contained either a different blue dye than that described above $(5 \mathrm{mg} / \mathrm{ml}$ erioglaucine), or 100 -fold more of the red dye than that used in our standard assays $(20 \mathrm{mg} / \mathrm{ml}$ sulforhodamine B). Flies were allowed to feed in the dark for $2 \mathrm{~h}$. As reported, the high concentration of red dye was repulsive, causing the flies to prefer the $5 \mathrm{~mm}$ sucrose plus erioglaucine (Mitri et al., 2009). To test for aversion to L-canavanine, we added $30 \mathrm{~mm} \mathrm{~L}$-canavanine to the $5 \mathrm{~mm}$ sucrose that contained the $5 \mathrm{mg} / \mathrm{ml}$ erioglaucine.

Electrophysiology. Tip recordings (Hodgson et al., 1955; Wieczorek and Wolff, 1989) were performed as described previously (Moon et al., 2006) using either $25 \mathrm{~mm}$ L-canavanine or $25 \mathrm{~mm}$ L-arginine. Briefly, newly eclosed flies were immobilized by inserting a glass capillary filled with Ringer's solution into the abdomen all the way to the head. This electrode also served as the indifferent electrode. The labellar hairs were stimulated with tastants dissolved in the buffer solution of the recording pipette (10-20 $\mu \mathrm{m}$ tip diameter). $\mathrm{KCl}$ at $1 \mathrm{~mm}$ was used as the electrolyte for all recordings. The recording electrode was connected to a preamplifier (TastePROBE, Syntech), and the signals were collected and amplified $(10 \times)$ using a signal connection interface box (Syntech) in conjunction with a $100-3000 \mathrm{~Hz}$ bandpass filter. Recordings of action potentials were acquired using a $12 \mathrm{kHz}$ sampling rate and analyzed using Autospike 3.1 software (Syntech).

Data analyses. All error bars represent SEMs. Unpaired Student's $t$ tests were used to compare two sets of data. ANOVA with the Tukey post hoc tests were used to compare multiple sets of data. Asterisks indicate statistical significance $\left({ }^{*} p<0.05,{ }^{* *} p<0.01\right)$.

\section{Results}

$m t t$ was dispensable for L-canavanine-induced action

potentials and avoidance

Mutation of the $m t t$ locus, which disrupts a GPCR (DmXR), has been reported to be necessary for the behavioral avoidance to L-canavanine (Mitri et al., 2009). In this prior study, the electrophysiological responses of gustatory receptor neurons were not tested. Therefore, we performed tip recordings to assess L-canavanine-induced action potentials. GRNs are housed in gustatory hairs (sensilla) situated on several body parts, including the main taste organ, the labellum, and are broadly grouped into three size classes [small (S); intermediate (I); large, (L)] (Vosshall and Stocker, 2007; Montell, 2009). We recorded from S3 sensilla, 
which respond to most aversive tastants (Weiss et al., 2011). In control flies, addition of L-canavanine stimulated action potentials $(10.6 \pm 1.9)$ immediately upon contact with the recording pipette (Fig. $1 A, B$ ). Surprisingly, we found that $m t t^{f 06268}$ mutant and wild-type flies displayed similar frequencies of L-canavanineinduced action potentials (Fig. $1 A, B$ ). Because $m t t^{f 06268}$ produced normal L-canavanine action potentials, we confirmed the reported transposon insertion in $m t t^{f 06268}$ by genomic PCR and analyzed $m t t^{f 06268}$ in trans with a deletion that completely removed $m t t, \mathrm{Df}(2 \mathrm{R})$ Exel7096. These latter flies also showed a wildtype electrophysiological response to L-canavanine (Fig. $1 B$ ).

Since the $m t^{f 06268} \mathrm{~S} 3$ sensilla responded normally to L-canavanine, we wondered whether other mutant sensilla might be impaired in L-canavanine-induced action potentials. To conduct this analysis, we first surveyed wild-type sensilla. Most I- and S-type sensilla, but none of the L-type sensilla, respond to aversive compounds (Weiss et al., 2011). Therefore, we recorded $\mathrm{L}$-canavanine-induced action potentials from the $10 \mathrm{~S}$-type sensilla (out of 12 total) that were most accessible for recordings. In addition, we sampled two I-type and two L-type sensilla, the latter of which we did not expect to be stimulated by $\mathrm{L}$-canavanine. The $\mathrm{S} 3, \mathrm{~S} 5$, and $\mathrm{S} 10$ sensilla were most responsive to L-canavanine ( $>10$ spikes/500 ms), while the S1, S6, S7, and S11 sensilla displayed a low-level activity (Fig. 1C). The remaining three S-type sensilla tested, S2, S4, and S8, were insensitive to L-canavanine (Fig. 1C). None of the L- or I-type sensilla examined were stimulated by L-canavanine (Fig. $1 C$ ). Given the high responsiveness of wild-type S5 and S10, we recorded from these latter sensilla from $m t t^{f 06268}$ and $m t t^{f 06268} / \mathrm{Df}$ flies and found that the responses were also indistinguishable from those of wild-type flies (Fig. $1 B$ ).

Due to the preceding results, we tested $m t^{f 06268}$ and $m t t^{f 06268} / \mathrm{Df}$ for behavioral avoidance to L-canavanine using a two-way choice behavioral assay (Meunier et al., 2003; Moon et al., 2006). We starved young flies for $24 \mathrm{~h}$ and allowed them to feed for $1.5 \mathrm{~h}$ in the dark in a microtiter dish that contained alternating wells consisting of $1 \mathrm{~mm}$ sucrose or $5 \mathrm{~mm}$ sucrose plus $30 \mathrm{~mm}$ L-canavanine. Each of the two tastants was mixed with red or blue food coloring. We then counted the number of flies with red, blue, or purple abdomens. Complete preferences for the 1 or $5 \mathrm{~mm}$ sucrose yield P.I.s of 1.0 and 0 respectively, while a P.I. of 0.5 results if there were a lack of bias for the two concentrations of sucrose. In the absence of L-canavanine, the control flies $\left(w^{1118}\right)$ selected the higher concentration of sucrose, and this preference was unaffected by the red or blue food coloring (P.I. $=0.07$ or 0.04 ; Fig. $1 \mathrm{D}$ ). When given a choice between $1 \mathrm{~mm}$ sucrose and 5 $\mathrm{mm}$ sucrose plus $30 \mathrm{mM}$ L-canavanine, the $w^{1118}$ flies decreased their preference for $5 \mathrm{~mm}$ sucrose (Fig. $1 D$ ). We assayed the behavioral responses to a range of L-canavanine concentrations. In control flies, $1 \mathrm{~mm} \mathrm{L-canavanine} \mathrm{did} \mathrm{not} \mathrm{affect} \mathrm{the} \mathrm{preference}$ for $5 \mathrm{~mm}$ sucrose over $1 \mathrm{~mm}$ sucrose, while 20 and $30 \mathrm{~mm}$ L-canavanine induced large avoidance responses (Fig. 1E). Consistent with the electrophysiological results, the $m t t^{f 06268}$ mutant displayed normal behavioral avoidance to L-canavanine. Wild-type and $m t t^{f 06268}$ flies also showed indistinguishable L-canavanine avoidance using a modified two-way choice test employed previously to characterize $m t t^{f 06268}$ (Fig. 1G; see Materials and Methods for detailed description). Based on the combination of electrophysiological and behavioral assays, we conclude that DmXR, which is encoded by $m t t$, was not required for detecting L-canavanine.
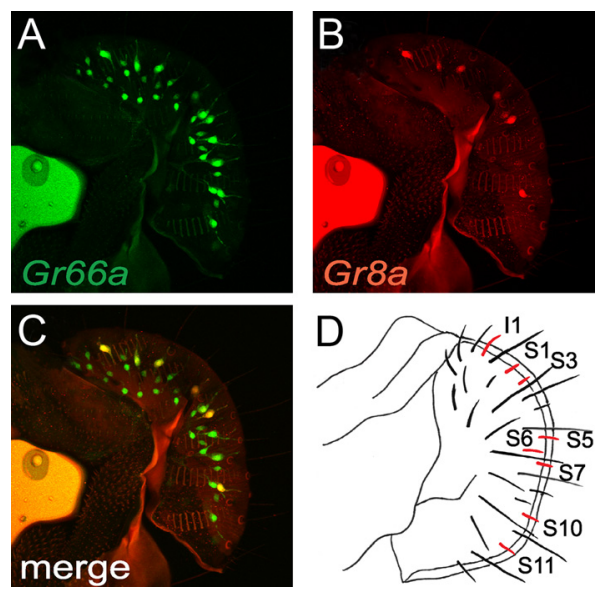

Figure 3. Expression of the Gr8a reporter in subsets of Gr66a-positive GRNs. A-C, Confocal images. A, Gr66a-I-GFP labeled all bitter responsive GRNs (anti-GFP, green). B, UAS-DsRed was expressed under control of the Gr8a-GAL4 (anti-DsRed, red). Most but not all Gr8a-postive cells were detected in single confocal images $\boldsymbol{C}$, Merged image of $\boldsymbol{A}$ and $\boldsymbol{B}$. D, Schematic illustration of a fly labellum showing gustatory sensilla that expressed the Gr8a reporter (red). The nomenclature for the sensilla is as described previously (Hiroi et al., 2002). This differs somewhat from another nomenclature (Weiss et al., 2011).

Requirement for GR8a and GR66a for avoiding L-canavanine To address whether a Drosophila GR was required for avoiding L-canavanine, we tested the available mutants that disrupt GRs that function in sensing aversive chemicals. These include Gr32a, Gr33a, Gr66a, Gr93a (Moon et al., 2006, 2009; Lee et al., 2009, 2010), and Gr47a (Y. Lee, S. J. Moon, and C. Montell, unpublished observations). In addition, we generated a null mutation in Gr8a by mobilizing a P-element that inserted in the second exon, thereby deleting most of the coding region (Fig. $2 A, B$ ). The $G r 8 a^{1}$ flies were homozygous viable and fertile.

We found that mutations disrupting $G r 8 a^{1}$ and $G r 66 a^{\text {ex83 }}$ severely impaired L-canavanine avoidance (Fig. $2 C$ ). These mutant flies displayed the same preference for $5 \mathrm{~mm}$ sucrose, even at the highest concentration of L-canavanine ( $30 \mathrm{mM}$; Fig. $2 \mathrm{D}$ ). Loss of the remaining four GRs that are known to affect the responses to aversive compounds had no impact on the repulsion to L-canavanine (Fig. 2C). These include Gr33a $a^{G A L 4}$, which is required for sensing all other noxious tastants tested (Moon et al., 2009), and $G r 32 a^{1}$ (Lee et al., 2010), which affected the responses to all avoidance tastants examined, except caffeine. We tested $G r 8 a^{1}$ using the previously described two-way choice test (Mitri et al., 2009) and found that the mutant was also impaired in L-canavanine repulsion using this alternative assay (Fig. 2 E).

To confirm that the deficits in L-canavanine avoidance were due to the mutations affecting $G r 8 a$ and $G r 66 a$, we tested for rescue of the mutant phenotypes with wild-type transgenes. Expression of UASGr8a under control of either the Gr66a promoter (Gr66a-GAL4) or the Gr8a promoter ( $G r 8 a-G A L 4)$ rescued the $G r 8 a^{l}$ phenotype (Fig. $2 F)$. Similarly, we rescued the Gr66 $a^{\text {ex83 }}$ phenotype using UASGr66 $a$ and either the Gr66a-GAL4 or the Gr8a-GAL4 (Fig. $2 F$ ). We detected expression of the Gr8a-GAL4 reporter in S-type sensilla (S1, S3, S5, S6, S7, S10, and S11) and one of I-type (I1) sensillum, but not other I- or L-type sensilla (Fig. 3), similar to that reported previously (Weiss et al., 2011).

\section{Elimination of L-canavanine-induced action potentials}

To test whether GR8a and GR66a were required for the electrophysiological responses to L-canavanine, we recorded from S3, S5, and S10 sensilla, which in control flies elicited the largest 
A
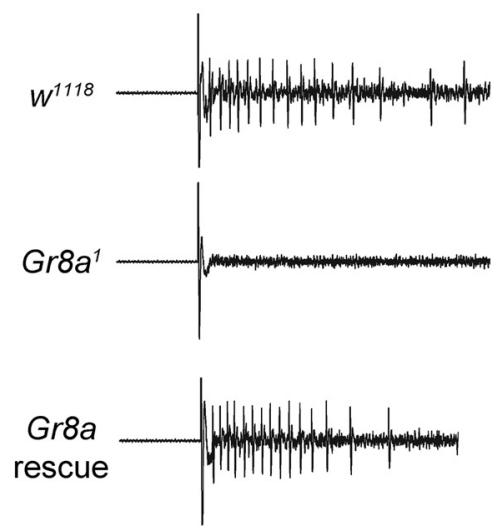

B
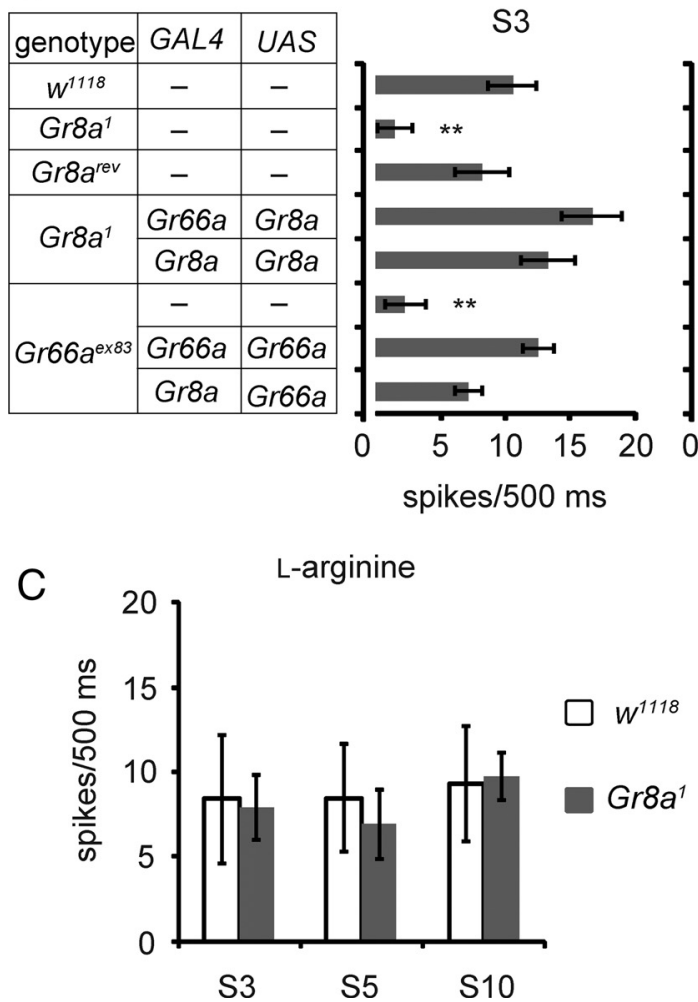

$$
\frac{1 \mathrm{mV} L}{50 \mathrm{~ms}}
$$

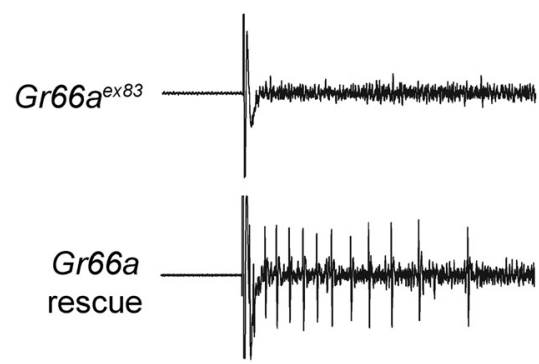

L-canavanine

S5

$\mathrm{S} 10$
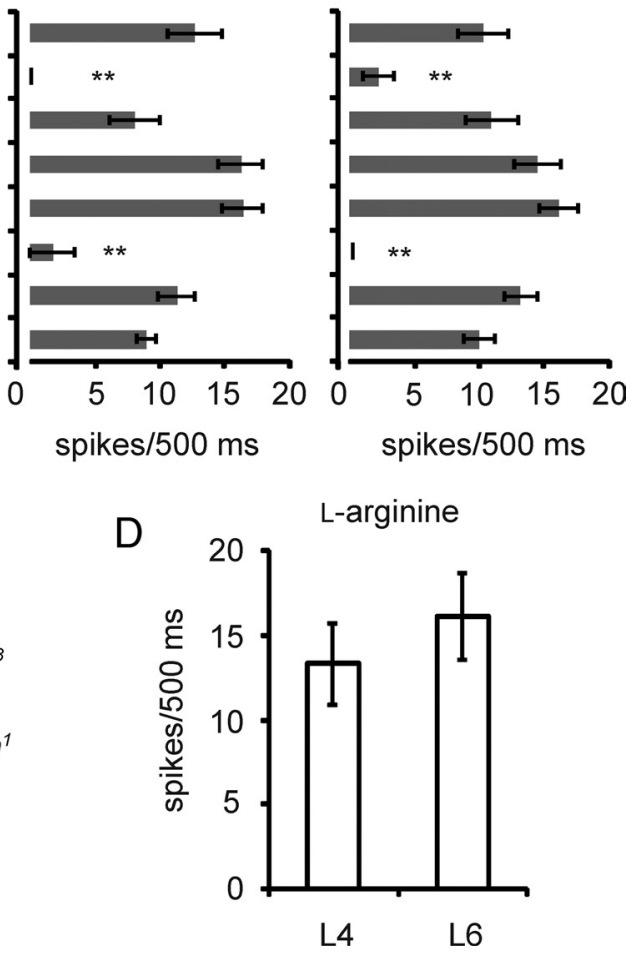

Figure 4. GR8a and GR66a are indispensible for L-canavanine-induced nerve firings. A, Representative traces of L-canavanine evoked action potentials from control flies $\left(w^{1118}\right)$, Gr8 $a^{1}$, Gr66a ${ }^{\text {ex83 }}$, and mutants with rescue transgenes (Gr8a'; Gr8a-GAL4/UAS-Gr8a and Gr8a-Gal4/UAS-Gr66a;Gr66a ${ }^{\text {ex83 }}$ ). The recordings were performed from S5 sensilla. B, Tip recordings showing that GR8a and GR66a are required for $\mathrm{L}$-canavanine-induced nerve firings. The assays were performed from $\mathrm{S3}$, S5, and S10 sensilla using animals of the indicted genotypes; $n=10-12$. The error bars represent SEMs; ${ }^{* *} p<0.01$. C, Tip recordings showing L-arginine-induced action potentials in S3, S5, and S10 sensilla; $n=10-11$. The error bars represent SEMs. D, $\mathrm{L}$-Arginine-induced action potentials in $\mathrm{L} 4$ and $\mathrm{L} 6$ sensilla; $n=10-11$. Shown are means \pm SEMs.

responses to L-canavanine. We found that L-canavanine-induced action potentials were eliminated in either the $G r 8 a^{1}$ or $G r 66 a^{\text {ex83 }}$ mutants and were restored upon expression of the wild-type transgenes that were driven under control of the Gr8a-GAL4 or Gr66a-GAL4 (Fig. 4A,B). The S3, S5, and S10 sensilla also responded to L-arginine, which is a structural analog of L-canavanine (Fig. 4C). L-Arginine evoked similar frequencies of action potentials in $G r 8 a^{1}$ as in wild type (Fig. $4 C$ ). L-type sensilla, which did not express the Gr8a reporter (Weiss et al., 2011), also responded to L-arginine (Fig. $4 D$ ).

\section{Distinct requirements for GR8a and GR66a}

Loss of GR66a diminishes the repulsion to caffeine, lobeline, and papaverine and the synthetic insect repellent DEET (Moon et al.,
2006; Lee et al., 2010). To investigate whether $G r 8 a^{1}$ displayed impaired avoidance to other noxious chemicals or was narrowly required for L-canavanine, we performed additional behavioral assays. $G r 8 a^{l}$ displayed normal responses to all other repulsive tastants tested: caffeine, quinine, berberine, denatonium, lobeline, papaverine, strychnine, and DEET (Fig. 5A). The frequencies of action potentials induced by application of these bitter chemicals were also similar between the mutant and the control flies (Fig. 5B). Thus, GR8a was more narrowly tuned to L-canavanine than GR66a.

\section{Discussion}

GRs but not DmXR are required for avoiding L-canavanine We conclude that GRs rather than the GPCR (DmXR) were essential for detecting the plant-derived toxin L-canavanine. Gr8a 

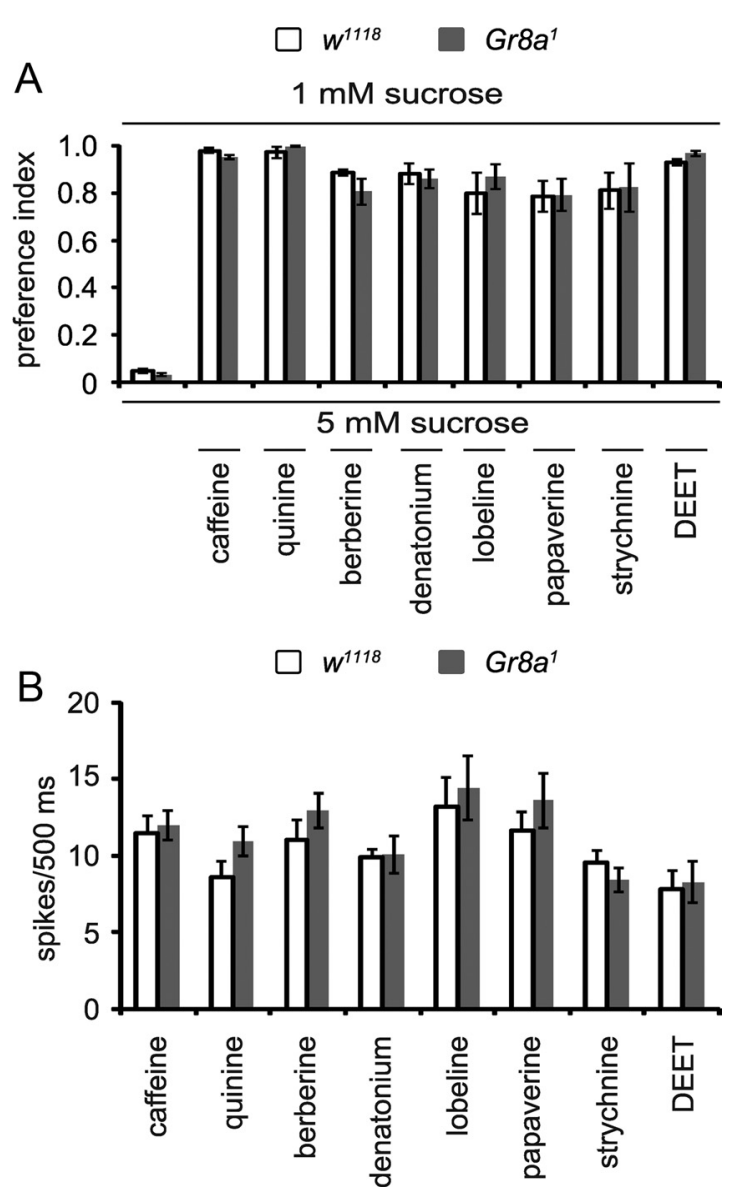

Figure 5. Behavioral and electrophysiological responses of $G r 8 a^{1}$ to multiple noxious tastants. A, Behavioral responses of $w^{1118}$ and $G r 8 a^{7}$ using two-way choice assays and one of the following concentrations of chemicals: $6 \mathrm{~mm}$ caffeine, $1 \mathrm{~mm}$ quinine, $0.05 \mathrm{~mm}$ berberine, $0.1 \mathrm{~mm}$ denatonium, $0.1 \mathrm{~mm}$ lobeline, $0.5 \mathrm{~mm}$ papaverine, $0.5 \mathrm{~mm}$ strychnine, or $0.2 \% \mathrm{DEET} ; n=5-12$. The error bars indicate SEMs. $\boldsymbol{B}$, Mean nerve firing responses from $w^{1118}$ and $G r 8 a^{1}$ by performing tip recordings from $S 6$ sensilla using the indicated concentrations of chemicals: $10 \mathrm{~mm}$ caffeine, $1 \mathrm{~mm}$ quinine, $0.1 \mathrm{~mm}$ berberine, $1 \mathrm{~mm}$ denatonium, $1 \mathrm{~mm}$ lobeline, $1 \mathrm{~mm}$ papaverine, 1 mu strychnine, $0.2 \%$ DEET; $n=11-18$. Shown are means \pm SEMs.

and Gr66a were required since mutations disrupting each of these genes eliminated the behavioral repulsion to the insecticide. These deficits were more dramatic than those associated with mutations disrupting the sensation of other aversive tastants, since avoidance to L-canavanine was abolished even at the highest concentrations examined. Both the Gr8a and Gr66a mutants displayed the same strong preference for $5 \mathrm{~mm}$ sucrose over $1 \mathrm{~mm}$ sucrose, even when the $5 \mathrm{~mm}$ sucrose was laced with $30 \mathrm{~mm}$ L-canavanine. Thus, in contrast to other aversive tastants that inhibit the sucrose response, thereby causing a reduced preference for $5 \mathrm{~mm}$ sucrose (Meunier et al., 2003), L-canavanine did not appear to inhibit the sugar response. In further support of the findings that Gr8a and Gr66a were required for sensing L-canavanine, we found that the electrophysiological responses to $\mathrm{L}$-canavanine were greatly reduced in the mutant flies $(\geq 7-10$ fold). Confirmation that Gr8a and Gr66a were essential for $\mathrm{L}$-canavanine repulsion was that we rescued the mutant phenotypes by introduction of wild-type transgenes.

In contrast to the requirements for Gr8a and Gr66a, the GPCR (DmXR) encoded by $m t t$ was dispensable for the repellent action of L-canavanine, since the behavior and electrophysiological responses of the mtt mutant were indistinguishable from those of control flies. This finding was not due to contamination of the $m t t^{f 06268}$ stock with wild-type flies, since we confirmed the presence of the $m t t$ mutation by PCR. The lack of requirement for DmXR described here was not due to differences in behavioral assays, since DmXR was also dispensable when we employed the two-way choice test used previously to assay $m \mathrm{tt}^{f 06268}$ mutant flies. Furthermore, we found that L-canavanine avoidance was not blocked with NMA, which has been reported to be a DmXR antagonist. Finally, we performed tip recordings and found that L-canavanine-induced action potentials depended on GR8a and GR66a and not on DmXR. Thus, while DmXR appears to be activated in vitro by L-canavanine, we conclude that GRs are the critical receptors for avoiding this plant-derived insecticide in vivo.

\section{Gr8a and Gr66a are corequired in S-type sensilla}

While Gr8a and Gr66a were both essential for avoiding L-canavanine, these two Grs displayed different expression patterns. Gr66a is widely expressed in most sensilla that respond to aversive compounds, which include the I-type sensilla, such as I7 and I8, which do not respond to L-canavanine (Hiroi et al., 2002; Thorne et al., 2004; Wang et al., 2004; Marella et al., 2006; Moon et al., 2006; Weiss et al., 2011). However, there was a strong correlation between sensilla that expressed Gr8a and displayed L-canavanine-induced activity. Sensilla with undetectable expression of the $G r 8 a$ reporter were unresponsive to L-canavanine (L-type, I-type, S2, S4, and S8), while the S-type sensilla with relatively high expression displayed L-canavanine-evoked action potentials. We were not able to record from the one I-type sensillum that expressed Gr8a (I1) due to poor accessibility of this sensillum. Gr66a and Gr8a were necessary for sensing L-canavanine in $\mathrm{Gr} 8 a$ expressing cells, since the loss of the L-canavanine response in the mutant flies was reversed by expression of the wild-type transgenes under control of the Gr8a promoter.

The requirements for GR8a and GR66a were also distinct. GR8a functioned narrowly in sensing L-canavanine. In contrast and consistent with the wider expression pattern of the Gr66a reporter, this latter GR is necessary for the repulsion to most (six of nine) noxious tastants examined (Moon et al., 2006; Lee et al., 2010). The other available $G r$ mutations did not affect L-canavanine repulsion. We suggest that GR8a and GR66a may form a multisubunit receptor that functions in a subset of S-type sensilla for L-canavanine repulsion. GR8a may contribute to the specificity for sensing L-canavanine, and GR66a may be a coreceptor for multiple GRs. However, GR8a and GR66a were not sufficient for responding to L-canavanine, since coexpression of the two GRs in tissue culture cells, ectopic coexpression of Gr8a and Gr66a in sugar-responsive GRNs, or Gr8a in Gr66aexpressing GRNs that did not express Gr8a (e.g., I7 or I8) did not confer L-canavanine sensitivity (data not shown).

\section{Aversive GRs comprised of subunits with narrow and broad specificities}

The widely differing specificities for the two GRs that act in sensing L-canavanine is reminiscent of our previous findings concerning the repertoire of GRs that function in caffeine repulsion. GR93a is required for avoidance of caffeine only (Lee et al., 2009), while mutations disrupting two other GRs that impair caffeine aversion (GR33a and GR66a) disrupt the repulsion to most deterrent tastants (Moon et al., 2006, 2009; Lee et al., 2009, 2010). Thus, while a single GR appears to be sufficient for the response to fructose (Sato et al., 2011), we propose that the GRs that participate in sensing noxious compounds are complexes comprised 
of at least one subunit with a narrow specificity and others that are broadly required coreceptors.

\section{References}

Brand AH, Perrimon N (1993) Targeted gene expression as a means of altering cell fates and generating dominant phenotypes. Development 118:401-415.

Clyne PJ, Warr CG, Carlson JR (2000) Candidate taste receptors in Drosophila. Science 287:1830-1834.

Dahanukar A, Lei YT, Kwon JY, Carlson JR (2007) Two Gr genes underlie sugar reception in Drosophila. Neuron 56:503-516.

Dahlman DL, Rosenthal GA (1975) Non-protein amino acid-insect interactions-I. Growth effects and symptomology of L-canavanine consumption by tobacco hornworm, Manduca sexta (L.). Comp Biochem Physiol A Comp Physiol 51:33-36.

Hiroi M, Marion-Poll F, Tanimura T (2002) Differentiated response to sugars among labellar chemosensilla in Drosophila. Zoolog Sci 19:1009-1018.

Hodgson ES, Lettvin JY, Roeder KD (1955) Physiology of a primary chemoreceptor unit. Science 122:417-418.

Jiao Y, Moon SJ, Montell C (2007) A Drosophila gustatory receptor required for the responses to sucrose, glucose, and maltose identified by mRNA tagging. Proc Natl Acad Sci U S A 104:14110-14115.

Jiao Y, Moon SJ, Wang X, Ren Q, Montell C (2008) Gr64f is required in combination with other gustatory receptors for sugar detection in Drosophila. Curr Biol 18:1797-1801.

Kang K, Pulver SR, Panzano VC, Chang EC, Griffith LC, Theobald DL, Garrity PA (2010) Analysis of Drosophila TRPA1 reveals an ancient origin for human chemical nociception. Nature 464:597-600.

Kim SH, Lee Y, Akitake B, Woodward OM, Guggino WB, Montell C (2010) Drosophila TRPA1 channel mediates chemical avoidance in gustatory receptor neurons. Proc Natl Acad Sci U S A 107:8440-8445.

Laski FA, Rio DC, Rubin GM (1986) Tissue specificity of Drosophila P element transposition is regulated at the level of mRNA splicing. Cell 44:7-19.

Lee Y, Moon SJ, Montell C (2009) Multiple gustatory receptors required for the caffeine response in Drosophila. Proc Natl Acad Sci U S A 106:4495-4500.

Lee Y, Kim SH, Montell C (2010) Avoiding DEET through insect gustatory receptors. Neuron 67:555-561.

Marella S, Fischler W, Kong P, Asgarian S, Rueckert E, Scott K (2006) Im- aging taste responses in the fly brain reveals a functional map of taste category and behavior. Neuron 49:285-295.

Meunier N, Marion-Poll F, Rospars JP, Tanimura T (2003) Peripheral coding of bitter taste in Drosophila. J Neurobiol 56:139-152.

Mitri C, Soustelle L, Framery B, Bockaert J, Parmentier ML, Grau Y (2009) Plant insecticide L-canavanine repels Drosophila via the insect orphan GPCR DmX. PLoS Biol 7:e1000147.

Miyamoto T, Amrein H (2008) Suppression of male courtship by a Drosophila pheromone receptor. Nat Neurosci 11:874-876.

Montell C (2009) A taste of the Drosophila gustatory receptors. Curr Opin Neurobiol 19:345-353.

Moon SJ, Köttgen M, Jiao Y, Xu H, Montell C (2006) A taste receptor required for the caffeine response in vivo. Curr Biol 16:1812-1817.

Moon SJ, Lee Y, Jiao Y, Montell C (2009) A Drosophila gustatory receptor essential for aversive taste and inhibiting male-to-male courtship. Curr Biol 19:1623-1627.

Robertson HM, Warr CG, Carlson JR (2003) Molecular evolution of the insect chemoreceptor gene superfamily in Drosophila melanogaster. Proc Natl Acad Sci U S A 100 [Suppl 2]:14537-14542.

Rosenthal GA (2001) L-Canavanine: a higher plant insecticidal allelochemical. Amino Acids 21:319-330.

Rosenthal GA, Dahlman DL (1986) L-Canavanine and protein synthesis in the tobacco hornworm Manduca sexta. Proc Natl Acad Sci U S A 83:14-18.

Sato K, Tanaka K, Touhara K (2011) Sugar-regulated cation channel formed by an insect gustatory receptor. Proc Natl Acad Sci U S A 108:11680-11685.

Thorne N, Chromey C, Bray S, Amrein H (2004) Taste perception and coding in Drosophila. Curr Biol 14:1065-1079.

Vosshall LB, Stocker RF (2007) Molecular architecture of smell and taste in Drosophila. Annu Rev Neurosci 30:505-533.

Wang Z, Singhvi A, Kong P, Scott K (2004) Taste representations in the Drosophila brain. Cell 117:981-991.

Weiss LA, Dahanukar A, Kwon JY, Banerjee D, Carlson JR (2011) The molecular and cellular basis of bitter taste in Drosophila. Neuron 69:258-272.

Wieczorek H, Wolff G (1989) The labellar sugar receptor of Drosophila. J Comp Physiol A 164:825-834.

Wisotsky Z, Medina A, Freeman E, Dahanukar A (2011) Evolutionary differences in food preference rely on Gr64e, a receptor for glycerol. Nat Neurosci 14:1534-1541. 